
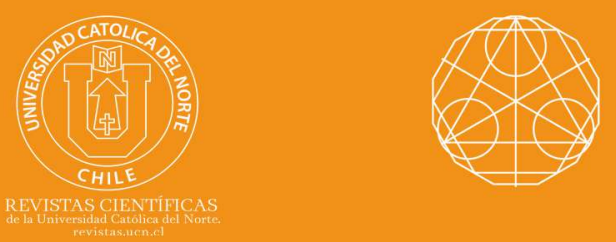

\title{
Analysis of Boundary value problem with multi- point conditions involving Caputo-Hadamard fractional derivative
}

Muthaiah Subramanian ${ }^{1}$ (1) orcid.org/0000-0001-5281-0935

Thangaraj Nandha Gopal ${ }^{2}$ (1) orcid.org/0000-0001-5475-9766

${ }^{1}$ KPR Institute of Engineering and Technology, Dept. of Mathematics, Coimbatore, TN, India. subramanianmcbe@gmail.com

${ }^{2}$ Sri Ramakrishna Mission Vidyalaya College of Arts and Science, Dept. of Mathematics, Coimbatore, TN, India.

nandhu792002@yahoo.co.in

Received: December 2018 | Accepted: March 2020

\section{Abstract:}

We study the boundary value problems (BVPs) of the Caputo-Hadamard type fractional differential equations (FDEs) supplemented by multi-point conditions. Many new results of existence and uniqueness are obtained with the use of fixed point theorems for single-valued maps. With the help of examples, the results are well illustrated.

Keywords: Caputo-Hadamard fractional derivative; Fractional differential equation; Hadamard fractional derivative; Hadamard fractional integral; Multi-point conditions; Existence; Fixed point.

MSC (2020): 26A33, 34A08, 34A12.

\section{Cite this article as (IEEE citation style):}

M. Subramanian and T. N. Gopal, "Analysis of Boundary value problem with multi-point conditions involving Caputo-Hadamard fractional derivative", Proyecciones (Antofagasta, On line) vol. 39, no. 6, pp. 1555-1575, Dec. 2020, doi: 10.22199/issn.0717-6279-2020-06-0093.

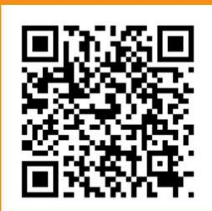

Article copyright: (C) 2020 Muthaiah Subramanian and Thangaraj Nandha Gopal. This is an open access article distributed under the terms of the Creative Commons License, which permits unrestricted use and distribution provided the original author and source are credited.

(cc) BY 


\section{Introduction}

Due to its applications in a few different disciplines of technical sciences and applied sciences, fractional calculus has gained significant importance in the last two decades. One of the main factors that represent the utility/prevalence of the subject is that fractional order derivatives and integrals are shown to be better devices for the representation of real components than integer orders. We refer the reader to the papers $[6,8,11$, $18,19,20,21,22,23,26,27,4,30,3,17]$ for examples and information, and the references cited therein. A large part of the work on the subject was found to depend on Riemann-Liouville and Caputo FDEs. Apart from Riemann-Liouville and Caputo derivatives, the collected works have other types of fractional derivatives, which are Hadamard [10], known as Hadamard derivatives and vary from the previous ones as the logarithmic function of the arbitrary exponent is included in their definition. A detailed description of Hadamard fractional derivative and integral can be found in $[2,1,7,14,16,29,28,25]$ and references cited therein. Note that the initial Hadamard type and BVPs are being investigated at their underlying level and need further consideration. Recently, in [15], Qinghua et.al discussed the inequality of Lyapunov-type with fractional Hadamard derivatives. Wang et.al [13] also studied Hadamard fractional BVP in nonlocal Hadamard with integral and discrete boundary conditions. The author has recently investigated FDEs involving Hadamard type derivatives with the nonlocal integral boundary conditions of the Hadamard type in [24]:

$$
\begin{aligned}
& D^{\alpha} u(t)+a(t) f(u(t))=0, \quad t \in[1, \infty] \\
& u(1)=0, \quad D^{\alpha-1} u(\infty)=\lambda_{i}{ }^{H} I^{\beta_{i}} u(\eta) .
\end{aligned}
$$

This study focuses on the existence and uniqueness of solutions to the following BVP of Caputo-Hadamard fractional-order differential equations (CHFDEs)

$$
\begin{aligned}
& { }^{C} D^{\zeta} q(t)=h(t, q(t)), \quad t \in[1, e], \\
& q(1)=0, \quad D^{\xi} q(\varphi)=\sum_{j=1}^{k-2} \varpi_{j} D^{\xi} q\left(\varrho_{j}\right), \\
& 1<\varphi<\varrho_{1}<\varrho_{2}<\cdots<\varrho_{k-2}<e,
\end{aligned}
$$

where ${ }^{C} D^{\zeta}$ denote the Caputo-Hadamard fractional derivative (CHFD) of order $1<\zeta \leq 2, D^{\xi}$ denote the Hadamard fractional derivative (HFD) of 
order $0<\xi<1$, and $h: J \times \mathbf{R} \rightarrow \mathbf{R}$ is given continuous function and $\varpi_{j}$ $(j=1,2, \ldots, k-2)$ are positive real constants. Here it should be emphasized that the multi-point conditions given by (1.2), is new, and can be construed as follows: the condition is proportional to the sum of their multi-point values with lower-order fractional derivative at the unknown point $(t=\varphi)$ of the interval with lower-order fractional derivative. Here we note that (1.2) consists of CHFDEs with nonlinearities involving unknown function and non-local multi-point boundary conditions. Whereas problem (1.1) deals with Hadamard FDEs with nonlinearities involving unknown function and nonlocal Hadamard type fractional integral boundary conditions. The rest of the paper is arranged as follows: the preliminary section covers some fundamental concepts of fractional calculus, with fundamental lemma linked to the given problem. The existence and uniqueness results are obtained from the nonlinear alternative Leray-Schauer, the Leray-Schauder Degree Theory, the fixed point theorems of Krasnoselskii, Schaefer, Banach, and Nonlinear contractions in Section 3. The results are validated by providing examples in Section 4.

\section{Preliminaries}

We start with some fundamental definitions, semigroup properties, and lemmas with results $[7,11]$.

DEFINITION 2.1.

Let $0 \leq b \leq c \leq \infty$ be finite or infinite interval of the half-axis $\mathbf{R}^{+}$. The Hadamard fractional integrals (HFIs) of order $\zeta \in \mathbf{C}$ are defined by

$$
\left(I_{b+}^{\zeta} h\right)(t)=\frac{1}{\Gamma(\zeta)} \int_{b}^{t}\left(\log \frac{t}{s}\right)^{\zeta-1} h(s) \frac{d s}{s}, \quad b<t<c
$$

and

$$
\left(I_{c-}^{\zeta} h\right)(t)=\frac{1}{\Gamma(\zeta)} \int_{t}^{c}\left(\log \frac{s}{t}\right)^{\zeta-1} h(s) \frac{d s}{s}, \quad b<t<c .
$$

Definition 2.2. The left and right-sided HFDs of order $\zeta \in \mathbf{C}$ with $\mathbf{R}(\zeta) \geq 0$ on $(b, c)$ and $b<t<c$ are defined by

$$
\left(D_{b+}^{\zeta} h\right)(t)=\left(t \frac{d}{d t}\right)^{n} \frac{1}{\Gamma(n-\zeta)} \int_{b}^{t}\left(\log \frac{t}{s}\right)^{n-\zeta-1} h(s) \frac{d s}{s}
$$


and

$$
\left(D_{c-}^{\zeta} h\right)(t)=\left(-t \frac{d}{d t}\right)^{n} \frac{1}{\Gamma(n-\zeta)} \int_{t}^{c}\left(\log \frac{s}{t}\right)^{n-\zeta-1} h(s) \frac{d s}{s},
$$

where $n=[\mathbf{R}(\zeta)]+1$.

If $\mathbf{R}(\zeta)>0, \mathbf{R}(\varsigma)>0$ and $0<b<c<\infty$, then we have

$$
\begin{aligned}
\left(I_{b+}^{\zeta}\left(\log \frac{s}{b}\right)^{\varsigma-1}\right)(t) & =\frac{\Gamma(\varsigma)}{\Gamma(\varsigma+\zeta)}\left(\log \frac{t}{b}\right)^{\varsigma+\zeta-1}, \\
\left(I_{c-}^{\zeta}\left(\log \frac{b}{s}\right)^{\varsigma-1}\right)(t) & =\frac{\Gamma(\varsigma)}{\Gamma(\varsigma+\zeta)}\left(\log \frac{c}{t}\right)^{\varsigma+\zeta-1}, \\
\left(D_{b+}^{\zeta}\left(\log \frac{s}{b}\right)^{\varsigma-1}\right)(t) & =\frac{\Gamma(\varsigma)}{\Gamma(\varsigma-\zeta)}\left(\log \frac{t}{b}\right)^{\varsigma-\zeta-1}, \\
\left(D_{c-}^{\zeta}\left(\log \frac{b}{s}\right)^{\varsigma-1}\right)(t) & =\frac{\Gamma(\varsigma)}{\Gamma(\varsigma-\zeta)}\left(\log \frac{c}{t}\right)^{\varsigma-\zeta-1} .
\end{aligned}
$$

Let $\zeta, \varsigma \in \mathbf{R}$ such that $\mathbf{R}(\zeta)>\mathbf{R}(\varsigma)>0$. If $0<b<c<\infty$ and $1 \leq p<\infty$, then for $h \in L^{p}(b, c)$,

$$
D_{b+}^{\varsigma} I_{b+}^{\zeta} h=I_{b+}^{\zeta-\varsigma} h \text { and } D_{c-}^{\varsigma} I_{c-}^{\zeta} h=I_{c-}^{\zeta-\varsigma} h .
$$

Definition 2.3. Let $0<b<c<\infty, \mathbf{R}(\zeta) \geq 0, n=[\mathbf{R}(\zeta)+1]$. The left and right CHFDs of order $\zeta$ are respectively defined by

$$
\left({ }^{C} D_{b+}^{\zeta} h\right)(t)=D_{b+}^{\zeta}\left[h(s)-\sum_{k=0}^{n-1} \frac{\delta^{k} h(b)}{k !}\left(\log \frac{s}{b}\right)^{k}\right](t),
$$

and

$$
\left({ }^{C} D_{c-}^{\zeta} h\right)(t)=D_{c-}^{\zeta}\left[h(s)-\sum_{k=0}^{n-1} \frac{(-1)^{k} \delta^{k} h(c)}{k !}\left(\log \frac{c}{s}\right)^{k}\right](t)
$$


Let $\zeta \geq 0$, and $n=[\zeta]+1$. If $h \in A C_{\delta}^{n}[b, c]$, where $0<b<c<\infty$. Then ${ }^{C} D_{b+}^{\zeta} h(t)$ and ${ }^{C} D_{c-}^{\zeta} h(t)$ exist everywhere on $[b, c]$ and

(a) if $\zeta \notin \mathbf{N}_{0}$,

$$
\begin{aligned}
& \left({ }^{C} D_{b+}^{\zeta} h\right)(t)=\frac{1}{\Gamma(n-\zeta)} \int_{b}^{t}\left(\log \frac{t}{s}\right)^{n-\zeta-1} \delta^{n} h(s) \frac{d s}{s} \\
& \left({ }^{C} D_{c-}^{\zeta} h\right)(t)=\frac{(-1)^{n}}{\Gamma(n-\zeta)} \int_{t}^{c}\left(\log \frac{s}{t}\right)^{n-\zeta-1} \delta^{n} h(s) \frac{d s}{s}
\end{aligned}
$$

(b) if $\zeta \in \mathbf{N}_{0}$, then

$$
\left({ }^{C} D_{b+}^{\zeta} h\right)(t)=\delta^{n} h(t), \quad\left({ }^{C} D_{c-}^{\zeta} h\right)(t)=(-1)^{n} \delta^{n} h(t) .
$$

In particular,

$$
\left({ }^{C} D_{b+}^{0} h\right)(t)=h(t), \quad\left({ }^{C} D_{c-}^{0} h\right)(t)=h(t) .
$$

Let $\zeta \geq 0$, and $n=[\zeta]+1$. If $v(t) \in A C_{\delta}^{n}[b, c]$, then the CHFDE

$$
{ }^{C} D_{b+}^{\zeta} v(t)=0
$$

has a solution:

$$
v(t)=\sum_{k=0}^{n-1} a_{k}\left(\log \frac{t}{b}\right)^{k}
$$

and the following formula holds:

$$
I_{b+}^{\zeta C} D_{b+}^{\zeta} v(t)=\sum_{k=0}^{n-1} a_{k}\left(\log \frac{t}{b}\right)^{k}
$$

where $a_{k} \in \mathbf{R}, k=1,2, \cdots n-1$.

We define space $Q=\{q(t): q(t) \in C([1, e], \mathbf{R})\}$ endowed with the norm $\|q\|=\sup \{|q(t)|, t \in[1, e]\}$. Obviously $(Q,\|\cdot\|)$ is a Banach space. Let $A C[1, e]$ be the space functions that are absolutely continuous on $[1, e]$. Let us introduce the space $A C_{\delta}^{n}[1, e]$, which consists of those functions $h$ by 


$$
A C_{\delta}^{n}[1, e]=\left\{h:[1, e] \rightarrow \mathbf{C}, \delta^{n-1} h(t) \in A C[1, e], \delta=t \frac{d}{d t}\right\}
$$

Given $\hat{h} \in C([1, e], \mathbf{R})$, the BVP

$$
\begin{aligned}
& { }^{C} D^{\zeta} q(t)=\hat{h}(t), \quad t \in[1, e], \\
& q(1)=0, \quad D^{\xi} q(\varphi)=\sum_{j=1}^{k-2} \varpi_{j} D^{\xi} q\left(\varrho_{j}\right), \\
& 1<\varrho_{1}<\varrho_{2}<\cdots<\varrho_{k-2}<e
\end{aligned}
$$

is equivalent to the integral equation

$$
q(t)=\frac{\log t}{\Theta}\left[{ }^{H} I^{\zeta-\xi} \hat{h}(\varphi)-\sum_{j=1}^{k-2} \varpi_{j}{ }^{H} I^{\zeta-\xi} \hat{h}\left(\varrho_{j}\right)\right]+{ }^{H} I^{\zeta} \hat{h}(t)
$$

with

$$
\Theta=\frac{1}{\Gamma(2-\xi)}\left[\sum_{j=1}^{k-2} \varpi_{j}\left(\log \varrho_{j}\right)^{1-\xi}-(\log \varphi)^{1-\xi}\right]
$$

\section{Existence Results : The Single-Valued Case}

In view of Lemma 2, we define an operator $F: Q \rightarrow Q$ as

$$
\begin{gathered}
F(q)(t)=\frac{\log t}{\Theta}\left[{ }^{H} I^{\zeta-\xi} h(s, q(s))(\varphi)-\sum_{j=1}^{k-2} \varpi_{j}{ }^{H} I^{\zeta-\xi} h(s, q(s))\left(\varrho_{j}\right)\right] \\
+{ }^{H} I^{\zeta} h(s, q(s))(t) .
\end{gathered}
$$

To run the interference for the proof, we introduce the notation:

$$
\Omega=\frac{1}{\Gamma(\zeta+1)}+\frac{1}{\Theta \Gamma(\zeta-\xi+1)}\left((\log \varphi)^{\zeta-\xi}+\sum_{j=1}^{k-2} \varpi_{j}\left(\log \varrho_{j}\right)^{\zeta-\xi}\right)
$$


Our first existence result is based on Leray-Schauder nonlinear alternative.

[9] Let $X$ be a Banach space, $E$ be a closed convex subset of $U, S$ an open subset of $E$ and $0 \in S$. Suppose that $F: \bar{S} \rightarrow E$ is a continuous, compact ((i.e.,) $F(\bar{S})$ is a relatively compact subset of $E$ ) map. Then either (i) $F$ has a fixed point in $\bar{S}$, or;

(ii) there is a $s \in \partial S$ (the boundary of $S$ in $E$ ) and $\nu \in(1, e)$ with $s=\nu F(s)$.

Assume that $h:[1, e] \times \mathbf{R} \rightarrow \mathbf{R}$ be a continuous function and the following conditions hold :

$\left(G_{1}\right)$ there exists a function $p \in C\left([1, e], \mathbf{R}^{+}\right)$, and $\phi: \mathbf{R}^{+} \rightarrow \mathbf{R}^{+}$nondecreasing such that

$$
|h(t, q)| \leq p(t) \phi(\|q\|) \text { for each }(t, q) \in[1, e] \times \mathbf{R}
$$

$\left(\mathrm{G}_{2}\right)$ there exists a number $M>0$ such that

$$
\begin{aligned}
\frac{M}{\|p\| \phi(M)} & >{ }^{H} I^{\zeta} p(s)(e)+H \\
H & =\frac{1}{\Theta}\left[{ }^{H} I^{\zeta-\xi} h(s, q(s))(\varphi)-\sum_{j=1}^{k-2} \varpi_{j}{ }^{H} I^{\zeta-\xi} h(s, q(s))\left(\varrho_{j}\right)\right] .
\end{aligned}
$$

Then, there exists at least one solution for the problem $(1.2)$ on $[1, e]$.

Proof. To begin with, the operator $F: Q \rightarrow Q$ is defined by (3.1). Next, we show that $F$ maps bounded sets into bounded sets in $C([1, e], \mathbf{R})$. For a positive number $\rho$, let $B_{\rho}=\{q \in C([1, e], \mathbf{R}):\|q\| \leq \rho\}$ be a bounded set in $C([1, e], \mathbf{R})$. Then, for each $q \in B_{\rho}$, we have

$$
\begin{aligned}
|(F q)(t)| \leq & \frac{\log t}{\Theta}\left[{ }^{H} I^{\zeta-\xi}|h(s, q(s))|(\varphi)+\sum_{j=1}^{k-2} \varpi_{j}{ }^{H} I^{\zeta-\xi}|h(s, q(s))|\left(\varrho_{j}\right)\right] \\
& +{ }^{H} I^{\zeta}|h(s, q(s))|(t) \\
\leq & \frac{\phi(\|q\|)}{\Theta}\left[{ }^{H} I^{\zeta-\xi} p(s)(\varphi)+\sum_{j=1}^{k-2} \varpi_{j}{ }^{H} I^{\zeta-\xi} p(s)\left(\varrho_{j}\right)\right]+\phi(\|q\|)^{H} I^{\zeta} p(s)(e),
\end{aligned}
$$


and consequently,

$\|F q\| \leq \phi(\|\rho\|) \frac{1}{\Theta}\left[{ }^{H} I^{\zeta-\xi} p(s)(\varphi)+\sum_{j=1}^{k-2} \varpi_{j}{ }^{H} I^{\zeta-\xi} p(s)\left(\varrho_{j}\right)\right]+\phi(\|\rho\|)^{H} I^{\zeta} p(s)(e)$.

We shall proceed to prove that the operator $F$ maps bounded sets into equicontinuous sets of $C([1, e], \mathbf{R})$. For $t_{1}, t_{2} \in[1, e]$ with $t_{1}<t_{2}$, and $q \in B_{\rho}$ is a bounded set of $C([1, e], \mathbf{R})$. Then we have

$$
\begin{aligned}
\left|(F q)\left(t_{2}\right)-(F q)\left(t_{1}\right)\right| \leq & \frac{\left|\log t_{2}-\log t_{1}\right|}{\Theta}\left[{ }^{H} I^{\zeta-\xi}|h(s, q(s))|(\varphi)\right. \\
& \left.+\sum_{j=1}^{k-2} \varpi_{j}{ }^{H} I^{\zeta-\xi}|h(s, q(s))|\left(\varrho_{j}\right)\right] \\
& +\left.\right|^{H} I^{\zeta}|h(s, q(s))|\left(t_{2}\right)-{ }^{H} I^{\zeta}|h(s, q(s))|\left(t_{1}\right) \mid \\
\leq & \frac{\left|\log t_{2}-\log t_{1}\right|}{\Theta}\left[{ }^{H} I^{\zeta-\xi} p(s)(\varphi)+\sum_{j=1}^{k-2} \varpi_{j}{ }^{H} I^{\zeta-\xi} p(s)\left(\varrho_{j}\right)\right] \\
& +\frac{\phi(\|\rho\|) \mid}{\Gamma(\zeta)} \mid \int_{0}^{t_{1}}\left[\left(\log \frac{t_{2}}{s}\right)^{\zeta-1}-\left(\log \frac{t_{1}}{s}\right)^{\zeta-1}\right] p(s) \frac{d s}{s} \\
& +\int_{t_{1}}^{t_{2}}\left(\log \frac{t_{2}}{s}\right)^{\zeta-1} p(s) \frac{d s}{s} \mid .
\end{aligned}
$$

Hence we have that right hand side of the above inequality tends to zero independent of $q \in B_{\rho}$ as $t_{2}-t_{1} \rightarrow 0$. Therefore, the operator $F(q)$ is equicontinuous and consequently, by Arzela-Ascoli theorem, it is completely continuous. Next, we show that the boundedness of the set of all solutions to equations $q=\nu F(q), 0<\nu<1$. Let $q$ be a solution. Then, for $t \in[1, e]$, and using the computations in proving that $F$ is bounded, we have

$$
\begin{aligned}
|(F q)(t)| \leq & \phi(\|q\|) \frac{1}{\Theta}\left[{ }^{H} I^{\zeta-\xi} p(s)(\varphi)+\sum_{j=1}^{k-2} \varpi_{j}{ }^{H} I^{\zeta-\xi} p(s)\left(\varrho_{j}\right)\right] \\
& +\phi(\|q\|)^{H} I^{\zeta} p(s)(e) \\
= & H+\phi(\|q\|)^{H} I^{\zeta} p(s)(e) .
\end{aligned}
$$


In view of $\left(G_{2}\right), \exists M \ni\|q\|=M$. Let us set

$$
L=\{q \in C([1, e], \mathbf{R}):\|q\|<M\} .
$$

Note that the operator $F: \bar{S} \rightarrow C([1, e], \mathbf{R})$ is continuous and completely continuous. From the of choice of $S$, there is no $q \in \partial S \ni q=$ $\nu F(q), 0<\nu<1$. Consequently, by the Lemma 3, we deduce that $F$ has a fixed point $q \in \bar{S}$ which is a solution of the problem (1.2).

Our second existence result is based on Leray-Schauder degree theory.

Let $h:[1, e] \times \mathbf{R} \rightarrow \mathbf{R}$ be a continuous function. Suppose that

$\left(G_{3}\right)$ there exists constants $0 \leq \varsigma<\Omega^{-1}$, and $P>0$ such that

$|h(t, q)| \leq \varsigma|q|+P \forall(t, q) \in[1, e] \times \mathbf{R}$,

where $\Omega$ is defined by (3.2). Then the BVP (1.2) has at least one solution on $[1, e]$.

Proof. We define an operator $F: Q \rightarrow Q$ as in (3.1). In view of the fixed point problem

$$
q=F q .
$$

We shall prove the existence of at least one solution $q \in C([1, e])$ satisfying (3.3). Set a ball $B_{R} \subset C([1, e])$, as

$$
B_{R}=\left\{q \in Q: \max _{t \in C([1, e])}|q(t)|<R\right\},
$$

with a constant radius $R>0$. Hence, we shall show that $F: \bar{B}_{R} \rightarrow C([1, e])$ satisfies a condition

$$
q=\tau F q, \quad \forall q \in \partial B_{R}, \quad \forall \tau \in[1, e] .
$$

We set

$$
F(\tau, q)=\tau F q, \quad q \in Q, \quad \tau \in[1, e] .
$$

As shown in Theorem 3 we have that the operator $F$ is continuous, uniformly bounded and equicontinuous. Then, by the Arzela-Ascoli theorem, a continuous map $g_{\tau}$ defined by $g_{\tau}(q)=q-F(\tau, q)=q-\tau F q$ is completely 
continuous. If (3.4) holds, then the following Leray-Schauder degrees are well defined and by the homotopy invariance of topological degree, it follows that

$$
\begin{aligned}
\operatorname{deg}\left(g_{\tau}, B_{R}, 0\right) & =\operatorname{deg}\left(I-\tau F, B_{R}, 0\right)=\operatorname{deg}\left(g, B_{R}, 0\right) \\
& =\operatorname{deg}\left(g_{0}, B_{R}, 0\right)=\operatorname{deg}\left(I, B_{R}, 0\right)=1=0, \quad 0 \in B_{R},
\end{aligned}
$$

where $I$ denotes the unit operator. By the nonzero property of LeraySchauder degree, $g_{1}(q)=q-F q=0$ for atleast one $q \in B_{R}$. Let us assume that $q=\tau F q$ for some $\tau \in[1, e]$ and for all $t \in[1, e]$ so that

$$
\begin{aligned}
|(F q)(t)| \leq & \frac{\log t}{\Theta}\left[{ }^{H} I^{\zeta-\xi}|h(s, q(s))|(\varphi)+\sum_{j=1}^{k-2} \varpi_{j}{ }^{H} I^{\zeta-\xi}|h(s, q(s))|\left(\varrho_{j}\right)\right] \\
& +{ }^{H} I^{\zeta}|h(s, q(s))|(t) \\
\leq & \varsigma|q|+P\left[\frac{1}{\Theta}\left({ }^{H} I^{\zeta-\xi} p(s)(\varphi)+\sum_{j=1}^{k-2} \varpi_{j}{ }^{H} I^{\zeta-\xi} p(s)\left(\varrho_{j}\right)\right)\right. \\
& \left.+{ }^{H} I^{\zeta} p(s)(e)\right] \\
= & (\varsigma|q|+P) \Omega,
\end{aligned}
$$

which, on taking norm $\sup _{t \in[1, e]}|q(t)|=\|q\|$ and solving for $\|q\|$, yields

$$
\|q\| \leq \frac{P \Omega}{1-\varsigma \Omega}
$$

If $R=\frac{\Omega}{1-\varsigma \Omega}+1$, inequality (3.4) holds.

Our third existence result is based on Krasnoselskii's fixed point theorem.

[12] Let $V$ be a closed convex and nonempty subset of a Banach space $X$. Let $F_{1}, F_{2}$ be the operators $\ni$

(i) $F_{1} q_{1}+F_{2} q_{2} \in V$ whenever $q_{1}, q_{2} \in V$;

(ii) $F_{1}$ is compact and continuous;

(iii) $F_{2}$ is a contraction mapping; Then there exists $q_{3} \in V$ such that $q_{3}=F_{1} q_{3}+F_{2} q_{3}$.

Let $h:[1, e] \times \mathbf{R} \rightarrow \mathbf{R}$ be a continuous function such that the following conditions hold: 
$\left(G_{4}\right) \quad\left|h\left(t, q_{1}\right)-h\left(t, q_{2}\right)\right| \leq W\left|q_{1}-q_{2}\right|, \forall t \in[1, e], q_{1}, q_{2} \in \mathbf{R}, W>0$.

$\left(G_{5}\right) \quad|h(t, q(t))| \leq \vartheta(t)$ for $(t, q) \in[1, e] \times \mathbf{R}$, and $\vartheta \in C\left([1, e], \mathbf{R}^{+}\right)$ with $\|\vartheta\|=\max _{t \in[1, e]}|\vartheta(t)|$.

If

$$
\left\{\frac{W}{\Theta}\left[\left(\frac{\sum_{j=1}^{k-2} \varpi_{j}\left(\log \varrho_{j}\right)^{\zeta-\xi}}{\Gamma(\zeta-\xi+1)}+\frac{(\log \varphi)^{\zeta-\xi}}{\Gamma(\zeta-\xi+1)}\right)\right]\right\}<1 .
$$

Then, there exists at least one solution for the problem $(1.2)$ on $[1, e]$.

Proof. Let us define $B_{\rho}=\{q \in Q:\|q\| \leq \rho\}$, where $\rho \geq\|\vartheta\| \Omega$. To prove the hypothesis of Lemma 3, we split the operator $F$ given by (3.1) as $F=F_{1}+F_{2}$ on $B_{\rho}$, where

$$
\begin{aligned}
\left(F_{1} q\right)(t) & ={ }^{H} I^{\zeta} h(s, q(s))(t), \\
\left(F_{2} q\right)(t) & =\frac{\log t}{\Theta}\left[{ }^{H} I^{\zeta-\xi} h(s, q(s))(\varphi)-\sum_{j=1}^{k-2} \varpi_{j}{ }^{H} I^{\zeta-\xi} h(s, q(s))\left(\varrho_{j}\right)\right] .
\end{aligned}
$$

For $\hat{q}_{1}, \hat{q}_{2} \in B_{\rho}$,

$$
\begin{aligned}
\left|\left(F_{1} \hat{q}_{1}\right)(t)+\left(F_{2} \hat{q}_{2}\right)(t)\right| \leq & \sup _{t \in[1, e]}\left\{\frac { \operatorname { l o g } t } { \Theta } \left[{ }^{H} I^{\zeta-\xi}|h(s, q(s))|(\varphi)\right.\right. \\
& \left.\left.+\sum_{j=1}^{k-2} \varpi_{j}{ }^{H} I^{\zeta-\xi}|h(s, q(s))|\left(\varrho_{j}\right)\right]+{ }^{H} I^{\zeta}|h(s, q(s))|(t)\right\} \\
\leq & \|\vartheta\|\left\{\frac{1}{\Theta}\left[\frac{(\log \varphi)^{\zeta-\xi}}{\Gamma(\zeta-\xi+1)}+\frac{\left(\sum_{j=1}^{k-2} \varpi_{j}\left(\log \varrho_{j}\right)^{\zeta-\xi}\right)}{\Gamma(\zeta-\xi+1)}\right]\right. \\
& \left.+\frac{1}{\Gamma(\zeta+1)}\right\} \\
\leq & \|\vartheta\| \Omega \leq \rho,
\end{aligned}
$$


which imply that $F_{1} \hat{q}_{1}+F_{2} \hat{q}_{2} \in B_{\rho}$.

Now, we will show that $F_{2}$ is a contraction. Let $q_{1}, q_{2} \in \mathbf{R}, t \in[1, e]$. Then, using the assumption $\left(G_{4}\right)$ together with (3.5), we get

$$
\left\|F_{2} q_{1}-F_{2} q_{2}\right\| \leq \frac{W}{\Theta}\left[\frac{\left(\sum_{j=1}^{k-2} \varpi_{j}\left(\log \varrho_{j}\right)^{\zeta-\xi}\right)}{\Gamma(\zeta-\xi+1)}+\frac{(\log \varphi)^{\zeta-\xi}}{\Gamma(\zeta-\xi+1)}\right]\left\|p_{1}-p_{2}\right\| .
$$

By the assumption $\left(G_{4}\right)$, it follows that the operator $F_{2}$ is contraction. Next, we will show that $F_{1}$ is compact and continuous. Continuity of $h$ implies that the operator $F_{1}$ is continuous. Also, $F_{1}$ is uniformly bounded on $B_{\rho}$ as

$$
\left\|F_{1} q\right\| \leq \frac{\|\vartheta\|}{\Gamma(\zeta+1)}
$$

Moreover, with $\sup _{(t, q) \in[1, e] \times B_{\rho}}|h(t, q)|=\hat{h}<\infty$ and $t_{1}<t_{2}, t_{1}, t_{2} \in[1, e]$, we have

$$
\begin{aligned}
\left|\left(F_{1} q\right)\left(t_{2}\right)-\left(F_{1} q\right)\left(t_{1}\right)\right|= & \left|{ }^{H} I^{\zeta}\right| h(s, q(s))\left|\left(t_{2}\right)-{ }^{H} I^{\zeta}\right| h(s, q(s))\left|\left(t_{1}\right)\right| \\
\leq & \frac{\hat{h}}{\Gamma(\zeta)} \mid \int_{0}^{t_{1}}\left[\left(\log \frac{t_{2}}{s}\right)^{\zeta-1}-\left(\log \frac{t_{1}}{s}\right)^{\zeta-1}\right] \frac{d s}{s} \\
& +\int_{t_{1}}^{t_{2}}\left(\log \frac{t_{2}}{s}\right)^{\zeta-1} \frac{d s}{s} \mid .
\end{aligned}
$$

Clearly, the right hand sides of (3.6) tends to zero independent of $q$ as $t_{2}-t_{1} \rightarrow 0$. Thus, $F_{1}$ is relatively compact on $B_{\rho}$. Hence, by the Arzela-Ascoli Theorem, $F_{1}$ is compact on $B_{\rho}$. Thus, all the assumptions of Lemma 3 are satisfied. Therefore, there exists at least one solution for problem $(1.2)$ on $[1, e]$.

Our next existence result is based on Schaefer's fixed point theorem.

[9] Let $X$ be a Banach space. Assume that $F: Q \rightarrow Q$ is a completely continuous operator and the set $A=\{q \in Q \mid q=\varepsilon F q, 0<\varepsilon<1\}$ is bounded. Then $F$ has a fixed point in $Q$.

Let $h:[1, e] \times \mathbf{R} \rightarrow \mathbf{R}$ be a continuous function. Assume that there exists a positive constant $\widehat{K}$ such that $|h(t, q)| \leq \widehat{K}$ for $t \in[1, e], p \in \mathbf{R}$. Then, there exists atleast one solution for problem $(1.2)$ on $[1, e]$. 
Proof. To begin with, we depict the operator $F: Q \rightarrow Q$ is completely continuous. By continuity of the function $h$, it follows that the operator $F$ is continuous.

For a positive constant $\rho$, let $B_{\rho}=\{q \in Q:\|q\| \leq \rho\}$ be a bounded set in $Q$. Then, for $t \in[1, e]$, we derive

$$
\begin{aligned}
|(F q)(t)| \leq & \frac{\log t}{\Theta}\left[\sum_{j=1}^{k-2} \varpi_{j}{ }^{H} I^{\zeta-\xi}|h(s, q(s))|\left(\varrho_{j}\right)+{ }^{H} I^{\zeta-\xi}|h(s, q(s))|(\varphi)\right] \\
& +{ }^{H}{ }^{\zeta}|h(s, q(s))|(t) \\
\leq & \widehat{K}\left\{\frac{1}{\Theta}\left[\sum_{j=1}^{k-2} \varpi_{j}{ }^{H} I^{\zeta-\xi}(1)\left(\varrho_{j}\right)+{ }^{H} I^{\zeta-\xi}(1)(\varphi)\right]+{ }^{H} I^{\zeta}(1)(e)\right\} \\
= & \widehat{K} \Omega .
\end{aligned}
$$

Hence it follows that $F$ is uniformly bounded. We shall proceed to prove that the operator $F$ is equicontinuous. For $t_{1}, t_{2} \in[1, e]$ with $t_{1}<t_{2}$, we have

$$
\begin{aligned}
\left|(F q)\left(t_{2}\right)-(F q)\left(t_{1}\right)\right| \leq & \frac{\left|\log t_{2}-\log t_{1}\right|}{\Theta}\left[{ }^{H} I^{\zeta-\xi}|h(s, q(s))|(\varphi)\right. \\
& \left.+\sum_{j=1}^{k-2} \varpi_{j}{ }^{H} I^{\zeta-\xi}|h(s, q(s))|\left(\varrho_{j}\right)\right] \\
& +{ }^{H} I^{\zeta}|h(s, q(s))|\left(t_{2}\right)-{ }^{H} I^{\zeta}|h(s, q(s))|\left(t_{1}\right) \\
\leq & \frac{\widehat{K}\left|\log t_{2}-\log t_{1}\right|}{\Theta}\left[{ }^{H} I^{\zeta-\xi}(\varphi)+\sum_{j=1}^{k-2} \varpi_{j}{ }^{H} I^{\zeta-\xi}\left(\varrho_{j}\right)\right] \\
& +\frac{\widehat{K}}{\Gamma(\zeta)} \mid \int_{0}^{t_{1}}\left[\left(\log \frac{t_{2}}{s}\right)^{\zeta-1}-\left(\log \frac{t_{1}}{s}\right)^{\zeta-1}\right] \frac{d s}{s} \\
& +\int_{t_{1}}^{t_{2}}\left(\log \frac{t_{2}}{s}\right)^{\zeta-1} \frac{d s}{s} \mid .
\end{aligned}
$$

Hence we have that right hand side of the above inequality tends to zero independent of $q \in B_{\rho}$ as $t_{2}-t_{1} \rightarrow 0$. Therefore, the operator $F(q)$ is equicontinuous and consequently, by Arzela-Ascoli theorem, it is completely 
continuous. Next, we consider the set $A=\{q \in Q: q=\varepsilon F(q), 0<\varepsilon<1\}$. Then, we have to show that $A$ is bounded, let $q \in A$ and $t \in[1, e]$. Then

$$
\begin{aligned}
\|q\| \leq & \widehat{K}\left\{\frac{1}{\Gamma(\zeta-\xi+1)}\left(\frac{1}{\Theta} \sum_{j=1}^{k-2} \varpi_{j}\left(\log \varrho_{j}\right)^{\zeta-\xi}\right)+\frac{(\log \varphi)^{\zeta-\xi}}{\Theta \Gamma(\zeta-\xi+1)}+\frac{1}{\Gamma(\zeta+1)}\right\} \\
= & \widehat{\Omega}
\end{aligned}
$$

Thus, $A$ is bounded. Hence it follows by Lemma 3 that the equation (1.2) has atleast one solution on $[1, e]$.

Next, we establish the uniqueness of solution using Banach fixed point theorem for problem (1.2).

Let $h:[1, e] \times \mathbf{R} \rightarrow \mathbf{R}$ be a continuous function satisfying the assumptions $\left(G_{4}\right)$. In addition, it is assumed that $W \Omega<1$, where $\Omega$ is defined by (3.2). Then, there exists a unique solution for problem $(1.2)$ on $[1, e]$.

Proof. Let us define $\sup _{t \in[1, e]}|h(t, 0)|=T<\infty$. Selecting $\rho \geq \frac{T \Omega}{1-W \Omega}$, we show that $F B_{\rho} \subset B_{\rho}$, where $B_{\rho}=\{q \in Q:\|q\| \leq \rho\}$. For $q \in B_{\rho}$, we have $\|(F q)(t)\|$

$$
\begin{aligned}
\leq & \sup _{t \in[1, e]}\left\{{ }^{H} I^{\zeta}|h(s, q(s))|(t)+\frac{\log t}{\Theta}\left[\sum_{j=1}^{k-2} \varpi_{j}{ }^{H} I^{\zeta-\xi}|h(s, q(s))|\left(\varrho_{j}\right)\right.\right. \\
& \left.\left.+{ }^{H} I^{\zeta-\xi}|h(s, q(s))|(\varphi)\right]\right\} \\
\leq & (W \rho+T) \sup _{t \in[1, e]}\left\{{ }^{H} I^{\zeta}(1) \mid(e)+\frac{1}{\Theta}\left[\sum_{j=1}^{k-2} \varpi_{j}{ }^{H} I^{\zeta-\xi}(1)\left(\varrho_{j}\right)+{ }^{H} I^{\zeta-\xi}(1)(\varphi)\right]\right\} \\
\leq & (W \rho+T) \Omega .
\end{aligned}
$$

Thus, it follows from (3.7) that $\|(F q)\| \leq \rho$.

Now, for $q, \hat{q} \in Q$, we derive 


$$
\begin{aligned}
|F q(t)-F \hat{q}(t)| \leq & \sup _{t \in[1, e]}\left\{\frac { \operatorname { l o g } t } { \Theta } \left[{ }^{H} I^{\zeta-\xi}|h(s, q(s))-h(s, \hat{q}(s))|(\varphi)\right.\right. \\
& \left.+\sum_{j=1}^{k-2} \varpi_{j}{ }^{H} I^{\zeta-\xi}|h(s, q(s))-h(s, \hat{q}(s))|\left(\varrho_{j}\right)\right] \\
& \left.+{ }^{H} I^{\zeta}|h(s, q(s))-h(s, \hat{q}(s))|(t)\right\} \\
\leq & {\left[\frac{W\|q-\hat{q}\|}{\Theta}\left({ }^{H} I^{\zeta-\xi}(\varphi)+\sum_{j=1}^{k-2} \varpi_{j}{ }^{H} I^{\zeta-\xi}\left(\varrho_{j}\right)\right)\right.} \\
& \left.+W\|q-\hat{q}\|^{H} I^{\zeta}(e)\right] \\
= & W \Omega\|q-\hat{q}\| .
\end{aligned}
$$

Thus,

$$
\|F q-F \hat{q}\| \leq W \Omega\|q-\hat{q}\| .
$$

Since $W \Omega<1$ by the given assumption, therefore $F$ is a contraction. Hence it follows by Banach fixed point theorem that the equation (1.2) has a unique solution on $[1, e]$.

Finally, we establish the uniqueness of solution using nonlinear contractions for problem (1.2).

Definition 3.1. Let $Q$ be a Banach space and let $F: Q \rightarrow Q$ be a mapping. $F$ is said to be a nonlinear contraction if there exists a continuous nondecreasing function $\Psi: \mathbf{R}^{+} \rightarrow \mathbf{R}^{+}$such that $\Psi(0)=0$ and $\Psi(\epsilon)<\epsilon$ for all $\epsilon>0$ with the property:

$$
\|F q-F \hat{q}\| \leq \Psi(\|q-\hat{q}\|), \quad \forall q, \hat{q} \in Q .
$$

(Boyd and Wong, [5]) Let $Q$ be a Banach space and let $F: Q \rightarrow Q$ be a nonlinear contraction. Then $F$ has a unique fixed point in $Q$. 
Let $h:[1, e] \times \mathbf{R} \rightarrow \mathbf{R}$ be a continuous function satisfying the assumption:

$\left(G_{6}\right) \quad|h(t, q)-h(t, \hat{q})| \leq g(t) \frac{|q-\hat{q}|}{\kappa+|q-\hat{q}|}, \forall t \in[1, e], q, \hat{q} \geq 0$, where $g:[1, e] \rightarrow \mathbf{R}^{+}$is continuous and $\kappa$ the constant defined by

$$
\kappa=\frac{1}{\Theta}\left[{ }^{H} I^{\zeta-\xi} g(\varphi)+\sum_{j=1}^{k-2} \varpi_{j}{ }^{H} I^{\zeta-\xi} g\left(\varrho_{j}\right)\right]+{ }^{H} I^{\zeta} g(e) .
$$

Then, there exists a unique solution for the problem $(1.2)$ on $[1, e]$.

Proof. Let us define the operator $F: Q \rightarrow Q$ as in (3.1) and the continuous nondecreasing function $\Psi: \mathbf{R}^{+} \rightarrow \mathbf{R}^{+}$by

$$
\Psi(v)=\frac{\kappa v}{\kappa+v}, \quad v>0 .
$$

Now, for $q, \hat{q} \in Q$ and for each $t \in[1, e]$, we derive

$$
\begin{aligned}
|F q(t)-F \hat{q}(t)| \leq & \sup _{t \in[1, e]}\left\{\frac { 1 } { \Theta } \left[{ }^{H} I^{\zeta-\xi}|h(s, q(s))-h(s, \hat{q}(s))|(\varphi)\right.\right. \\
& \left.+\sum_{j=1}^{k-2} \varpi_{j}{ }^{H} I^{\zeta-\xi}|h(s, q(s))-h(s, \hat{q}(s))|\left(\varrho_{j}\right)\right] \\
& \left.+{ }^{H} I^{\zeta}|h(s, q(s))-h(s, \hat{q}(s))|(t)\right\} \\
\leq & {\left[\frac { 1 } { \Theta } \left({ }^{H} I^{\zeta-\xi}\left(g(s) \frac{|q-\hat{q}|}{\Psi+|q-\hat{q}|}\right)(\varphi)\right.\right.} \\
& \left.+\sum_{j=1}^{k-2} \varpi_{j}{ }^{H} I^{\zeta-\xi}\left(g(s) \frac{|q-\hat{q}|}{\Psi+|q-\hat{q}|}\right)\left(\varrho_{j}\right)\right) \\
& \left.+{ }^{H} I^{\zeta}\left(g(s) \frac{|q-\hat{q}|}{\Psi+|q-\hat{q}|}\right)(e)\right] \\
\leq & \frac{\Psi\|q-\hat{q}\|}{\kappa}\left[\frac{1}{\Theta}\left({ }^{H} I^{\zeta-\xi} g(\varphi)+\sum_{j=1}^{k-2} \varpi_{j}{ }^{H} I^{\zeta-\xi} g\left(\varrho_{j}\right)\right)+{ }^{H} I^{\zeta} g(e)\right] \\
= & \Psi\|q-\hat{q}\| .
\end{aligned}
$$


This implies that, $\|F q-F \hat{q}\| \leq \Psi(\|q-\hat{q}\|)$. Therefore $F$ is a nonlinear contraction. Hence it follows by nonlinear contractions that the equation (1.2) has a unique solution on $[1, e]$.

\section{Examples}

EXAmple 4.1. Consider the following fractional-order BVP

$$
{ }^{C} D^{\frac{7}{5}} q(t)=\frac{\sqrt{5}}{3}+\frac{|q(t)|}{1+|q(t)|} \cdot \frac{1}{(2+\log t)^{2}}, \quad t \in[1, e],
$$

subject to the multi-point boundary conditions

$$
q(1)=0, \quad D^{\frac{3}{4}} q(\varphi)=\sum_{j=1}^{k-2} \varpi_{j} D^{\frac{3}{4}} q\left(\varrho_{j}\right) .
$$

Here, $\zeta=\frac{7}{5}, \xi=\frac{3}{4}, k=5, \varphi=2, \varpi_{1}=\frac{1}{10}, \varpi_{2}=\frac{1}{3}, \varpi_{3}=\frac{1}{2}, \varrho_{1}=\frac{5}{2}$, $\varrho_{2}=\frac{7}{3}, \varrho_{3}=\frac{9}{4}$. In addition, we find that

$$
\begin{aligned}
|h(t, q(t))| & =\frac{\sqrt{5}}{3}+\frac{|q(t)|}{1+|q(t)|} \cdot \frac{1}{(2+\log t)^{2}} \quad \text { as } \\
\left|h\left(t, q_{1}(t)\right)-h\left(t, q_{2}(t)\right)\right| & \leq \frac{1}{9}\left\|q_{1}-q_{2}\right\| .
\end{aligned}
$$

With the given data, we find that $\Theta=1.6162109015132577, \Omega=$ 1.4518469276542298. Thus, $W \Omega \cong 0.1613163252949144<1$, all the assumptions of Theorem 3 are satisfied. Hence, by Theorem 3, the BVP (4.1)-(4.2) has a unique solution on $[1, e]$.

EXAmple 4.2. Consider the following fractional-order BVP

$$
{ }^{C} D^{\frac{5}{3}} q(t)=1+\frac{|q(t)|}{1+|q(t)|} \cdot \frac{\log t}{4+\log t}, \quad t \in[1, e],
$$

subject to the multi-point boundary conditions of Example 4.1.

Here, $\zeta=\frac{5}{3}, \xi=\frac{3}{4}, k=5, \varphi=2, \varpi_{1}=\frac{1}{10}, \varpi_{2}=\frac{1}{3}, \varpi_{3}=\frac{1}{2}, \varrho_{1}=\frac{5}{2}$, $\varrho_{2}=\frac{7}{3}, \varrho_{3}=\frac{9}{4}$. In addition, we find that

$$
\begin{aligned}
|h(t, q(t))| & =1+\frac{|q(t)|}{1+|q(t)|} \cdot \frac{\log t}{4+\log t} \quad \text { as } \\
\left|h\left(t, q_{1}(t)\right)-h\left(t, q_{2}(t)\right)\right| & \leq \frac{1}{5}\left\|q_{1}-q_{2}\right\| .
\end{aligned}
$$


With the given data, we find that $\Theta=1.6162109015132577, \Omega=$ 1.112952553026304 .

Thus, $\left\{\frac{W}{\Theta}\left[\left(\frac{\sum_{j=1}^{k-2} \varpi_{j}\left(\log \varrho_{j}\right)^{\zeta-\xi}}{\Gamma(\zeta-\xi+1)}+\frac{(\log \varphi)^{\zeta-\xi}}{\Gamma(\zeta-\xi+1)}\right)\right]\right\} \cong 0.08966265051336413$, all the assumptions of Theorem 3 are satisfied. Hence, by Theorem 3, the BVP (4.3) with (4.2) has at least one solution on $[1, e]$.

\section{References}

[1] B. Ahmad and S. K. Ntouyas, "Initial value problems for functional and neutral functional Hadamard type fractional differential inclusions", Miskolc mathematical notes, vol. 17, no. 1, pp. 15-27, 2016, doi: 10.18514/ MMN.2016.1632

[2] B. Ahmad, SK. Ntouyas, R. P. Agarwal, and A. Alsaedi, "New results for boundary value problems of Hadamard-type fractional differential inclusions and integral boundary conditions", Boundary value problems, Art. ID. 275, Dec. 2013, doi: 10.1186/ 1687-2770-2013-275

[3] A. Ali, K. Shah, F. Jarad, V. Gupta, and T. Abdeljawad, "Existence and stability analysis to a coupled system of implicit type impulsive boundary value problems of fractional-order differential equations", Advances in difference equations, Art. ID. 101, Mar. 2019, doi: 10.1186/ s13662-019-2047-y

[4] Z. Ali, A. Zada, and K. Shah, "Existence and stability analysis of three point boundary value problem", International journal of applied and computational mathematics, vol. 3, no. 1, pp. 651-664, 2017, doi : $10.1007 /$ s40819-017-0375-8

[5] D. W. Boyd and J. S. W. Wong, "On nonlinear contractions", Proceedings of the American Mathematical Society, vol. 20, no. 2, pp. 458-464, 1969, doi: $10.2307 / 2035677$ 
[6] P. Duraisamy, A. R. Vidhya Kumar, T. Nandha Gopal, and M. Subramanian, "Influence of nonlocal discrete and integral boundary conditions involving Caputo derivative in boundary value problem", Journal of physics: conference series, vol. 1139, no. 1, Art. ID. 012014, 2018, doi: 10.1088/ 1742-6596/ 1139/1/ 012014

[7] Y. Y. Gambo, F. Jarad, D. Baleanu, and T. Abdeljawad, "On Caputo modification of the Hadamard fractional derivatives", Advances in difference equations, Art. ID. 10, 2014, doi: 10.1186/1687-18472014-10

[8] A. Guezane-Lakoud, G. Rebiai, and R. Khaldi, "Existence of solutions for a nonlinear fractional system with nonlocal boundary conditions", Proyecciones (Antofagasta. On line), vol. 36, no. 4, pp. 727-737, 2017, doi: 10.4067/ S0716-09172017000400727

[9] A. Granas and J. Dugundji, Fixed point theory, New York, NY : Springer, 2003, doi: 10.1007/ 978-0-387-21593-8

[10] J. Hadamard, "Essai sur letude des fonctions donnees par leur development de Taylor", Journal de mathématiques pures et appliquées 4e série, vol. 8, pp. 101-186, 1892. [On line]. Available: https:/ / bit.ly/ 3eqjKIN

[11] A. A. Kilbas, H. M. Srivastava, and J. J. Trujillo, Theory and applications of fractional differential equations, Amsterdam: Elsevier, 2006.

[12] M. A. Krasnoselskii, "Two remarks on the method of successive approximations", Uspekhi matematicheskikh nauk, vol. 10, no. 1, pp. 123-127, 1955. [On line]. Available: https:/ / bit.ly/ 32cLcF9

[13] S. Muthaiah, M. Murugesan, and N. Thangaraj, "Existence of solutions for nonlocal boundary value problem of Hadamard fractional differential equations", Advances in the theory of nonlinear analysis and its applications, vol. 3, no. 3, pp. 162-173, 2019, doi: $10.31197 /$ atnaa. 579701

[14] I. Podlubny, Fractional differential equations: an introduction to fractional derivatives, fractional differential equations, some methods of their solution and some of their applications, New York, NY: Academic Press, 1999.

[15] M. Qinghua, M. Chao, and J. Wang, "A Lyapunov-type inequality for a fractional differential equation with Hadamard derivative", Journal of mathematical inequalities, vol. 11, no. 1, pp. 135-141, 2017, doi: 10.7153/jmi-11-13 
[16] J. Sabatier, O. P. Agrawal, and J. A. Tenreiro Machado, Advances in fractional calculus: theoretical developments and applications in physics and engineering, Dordrecht: Springer, 2007, doi: 10.1007/978-1-4020-6042-7

[17] K. Shah, A. Ali, and S. Bushnaq, "Hyers-Ulam stability analysis to implicit Cauchy problem of fractional differential equations with impulsive conditions", Mathematical methods in the applied sciences, vol. 41, no. 17, pp. 8329-8343, 2018, doi: 10.1002/ mma.5292

[18] M. Subramanian, A. R. Vidhya Kumar, and T. Nandha Gopal, "Analysis of fractional boundary value problem with non local flux multipoint conditions on a Caputo fractional differential equation", Studia Universitatis Babes-Bolyai Mathematica, vol. 64, no. 4, pp. 511-527, 2019, doi : 10.24193/ subbmath.2019.4.06

[19] M. Subramanian, A. R. Vidhya Kumar, and T. Nandha Gopal, "A fundamental approach on non-integer order differential equation using nonlocal fractional sub-strips boundary conditions", Discontinuity, nonlinearity, and complexity, vol. 8, no. 2, pp. 189-199, 2019, doi: 10.5890/ DNC.2019.06.006

[20] M. Subramanian, A. R. Vidhya Kumar, and T. Nandha Gopal, "Analysis of fractional boundary value problem with non-local integral strip boundary conditions", Nonlinear studies, vol. 26, no. 2, pp. 445-454, 2019. [On line]. Available: https:/ / bit.ly/ 2JrSVso

[21] M. Subramanian, A. R. Vidhya Kumar, and T. Nandha Gopal, "Influence of coupled nonlocal slit-strip conditions involving Caputo derivative in fractional boundary value problem", Discontinuity, nonlinearity, and complexity, vol. 8, no. 4, pp. 429-445, 2019, doi: 10.5890/ DNC.2019.12.007

[22] M. Subramanian, A. R. Vidhya Kumar, and T. Nandha Gopal, "A writ large analysis of complex order coupled differential equations in the ourse of coupled non-local multi-point boundary conditions", Advanced studies in contemporary mathematics, vol. 29, no. 4, pp. 505-520, 2019, doi: 10.17777/ ascm2019.29.4.505

[23] M. Subramanian, A. R. Vidhya Kumar, and T. Nandha Gopal, "A strategic view on the consequences of classical integral sub-strips and coupled nonlocal multi-point boundary conditions on a combined Caputo fractional differential equation", Proceedings of the Jangjeon Mathematical Society, vol. 22, no. 3, pp. 437-453, 2019, doi: 10.17777/ pjms2019.22.3.437 
[24] P. Thiramanus, S. K. Ntouyas, and J. Tariboon, "Positive solutions for Hadamard fractional differential equations on infinite domain", Advances in difference equations, Art. ID. 83, 2016, doi: 10.1186/ s13662-016-0813-7

[25] E. Ugurlu, D. Baleanu, and K. Tas, "On the solutions of a fractional boundary value problem", Turkish journal of mathematics, vol. 42, no. 3, pp. 1307-1311, 2018, doi: 10.3906/ mat-1609-64

[26] A. R. Vidhya Kumar, P. Duraisamy, T. Nandha Gopal and M. Subramanian, "Analysis of fractional differential equation involving Caputo derivative with nonlocal discrete and multi-strip type boundary conditions", Journal of physics: conference series, vol. 1139, no. 1, pp. 012020, 2018, doi: 10.1088/ 1742-6596/ 1139/ 1/ 012020

[27] G. Wang, K. Pei, R. P. Agrawal, L. Zhang, and B. Ahmad, "Nonlocal Hadamard fractional boundary value problem with Hadamard integral and discrete boundary conditions on a half-line", Journal of computational and applied mathematics, Vol. 343, pp. 230-239, 2018, doi: 10.1016/j.cam.2018.04.062

[28] T. A. Yildiz, N. Khodabakhshi and D. Baleanu, "Analysis of mixedorder Caputo fractional system with nonlocal integral boundary condition", Turkish journal of mathematics, vol. 42, no. 3, pp. 1328-1337, 2018, doi: 10.3906/ mat-1709-71

[29] N. Yukunthorn, B. Ahmad, SK. Ntouyas, and J. Tariboon, "On CaputoHadamard type fractional impulsive hybrid systems with nonlinear fractional integral conditions", Nonlinear analysis: hybrid systems, vol. 19, pp. 77-92, 2016, doi: 10.1016/j.nahs.2015.08.001

[30] A. Zada, R. Rizwan, J. Xu, and Z. Fu, "On implicit impulsive Langevin equation involving mixed order derivatives", Advances in difference equations, Art. ID. 489, 2019, doi: 10.1186/ s13662-019-2408-6 